\title{
Hepatocyte selection medium-enriched hepatocellular carcinoma cells are positive for $\alpha$-fetoprotein and CD44
}

\author{
MINORU TOMIZAWA ${ }^{1}$, FUMINOBU SHINOZAKI ${ }^{2}$, YASUFUMI MOTOYOSHI ${ }^{3}$, \\ TAKAO SUGIYAMA $^{4}$, SHIGENORI YAMAMOTO ${ }^{5}$ and NAOKI ISHIGE ${ }^{6}$ \\ Departments of ${ }^{1}$ Gastroenterology, ${ }^{2}$ Radiology, ${ }^{3}$ Neurology, ${ }^{4}$ Rheumatology, ${ }^{5}$ Pediatrics and ${ }^{6}$ Neurosurgery, \\ National Hospital Organization, Shimoshizu Hospital, Yotsukaido, Chiba 284-0003, Japan
}

Received May 4, 2016; Accepted March 3, 2017

DOI: $10.3892 / \mathrm{ol} .2017 .6239$

\begin{abstract}
Tissues surrounding hepatocellular carcinomas (HCCs) lack glucose. Hepatocyte selection medium (HSM) is deficient in glucose and is supplemented with galactose. HCC cells were cultured in HSM to investigate the stem cell markers $\alpha$-fetoprotein (AFP) and cluster of differentiation 44 (CD44). HCC cells (HLF and PLC/PRF/5 cells) were cultured in HSM. Viable cell numbers were determined on days 0 and 7 following culture in HSM. RNA was isolated and subjected to reverse transcription-quantitative PCR (RT-qPCR) to analyze the mRNA expression levels of AFP and CD44. Immunostaining was performed to analyze the protein levels of AFP and CD44. The number of viable cells was significantly decreased on day 7 following culture in HSM. The expression levels of AFP and CD44 increased on day 7 as assessed using RT-qPCR. Immunostaining confirmed the results of RT-qPCR analysis. The number of viable HCC cells was decreased in HSM, whereas the expression levels of AFP and CD44 increased. Therefore, HSM is potentially useful for the enrichment of HCC cells with cancer stem cell characteristics.
\end{abstract}

\section{Introduction}

Hepatocellular carcinoma (HCC) occurs in the liver and is related to infection by hepatitis B or C virus (1). HCC cells maintain their proliferative potential in fibrous tissues with limited amounts of glucose and arginine (2), despite glucose being indispensable for cell survival (3). Hepatocytes produce energy from galactose via glycolysis $(4,5)$ and arginine is an essential amino acid in this process (6). Hepatocytes produce arginine from ornithine via the urea cycle. Hepatocyte

Correspondence to: Dr Minoru Tomizawa, Department of Gastroenterology, National Hospital Organization, Shimoshizu Hospital, 934-5 Shikawatashi, Yotsukaido, Chiba 284-0003, Japan E-mail: nihminor-cib@umin.ac.jp

Key words: $\alpha$-fetoprotein, cluster of differentiation 44, immunostaining, reverse transcription-quantitative polymerase chain reaction, glucose, arginine selection medium (HSM) is deficient in glucose and arginine, and is supplemented with galactose and ornithine (7). HSM enriches hepatocytes from co-culture with human induced pluripotent stem cells (8). HSM is also applicable to a condition similar to fibrous tissues (9).

$\alpha$-fetoprotein (AFP) is a marker of immature hepatocytes (10). HCC cells are immature hepatocytes and produce AFP $(11,12)$. CD44 is a receptor of hyaluronan (13) and is used as a marker of cancer stem cells (14). In a previous study, CD44 was not identified to be expressed in surgical specimens of HCC because the presence of cancer stem cells is rare (15). In another previous study, HCC cells were demonstrated to be heterogeneously positive for AFP and CD44 (16). These studies suggest that the positivity of CD44 in HCC cells remains controversial. Alterations in the expression patterns of AFP and CD44 are not known in HCC cells cultured without glucose or arginine.

Therefore, in the present study, the expression patterns of AFP and CD44 were investigated in HCC cells cultured in HSM to examine the alterations in the characteristics of HCC cells grown in the absence of glucose and arginine.

\section{Materials and methods}

Cell culture and determination of cell numbers. The HCC cell lines HLF and PLC/PRF/5 cells (RIKEN Cell Bank, Tsukuba, Japan) were cultured in Dulbecco's modified Eagle's medium (DMEM) supplemented with $10 \%$ fetal bovine serum (Thermo Fisher Scientific, Inc., Waltham, MA, USA) in 6-well plates (AGC Techno Glass Co., Ltd., Shizouka, Japan) at $37^{\circ} \mathrm{C}$ in a humidified chamber containing $5 \% \mathrm{CO}_{2}$. Cells $\left(1 \times 10^{4}\right)$ were spread onto each well of the 6 -well plates, and viable cell numbers were determined on days 0 and 7 following culture using the trypan blue dye exclusion test.

Hepatocyte selection medium. HSM was prepared from amino acid powders, using the formulation of Leibovitz L-15 medium (Thermo Fisher Scientific, Inc.), omitting arginine, tyrosine, glucose and sodium pyruvate, but with the addition of $900 \mathrm{mg} / \mathrm{l}$ galactose, $1 \mathrm{mM}$ ornithine, $5 \mathrm{mM}$ glycerol and $260 \mathrm{mM}$ proline (all Wako Pure Chemical Industries, Osaka, Japan). Proline was included in the medium as it is required for DNA synthesis (17). Knockout serum replacement (Thermo Fisher Scientific, Inc.) 
Table I. Sequences of primers used in the quantitative polymerase chain reaction.

\begin{tabular}{lllcc}
\hline Primer name & \multicolumn{1}{c}{ Sequence } & \multicolumn{1}{c}{ Description } & Product size (bp) & GenBank ${ }^{\circledR}$ accession no. \\
\hline OMC317 & 5'-ACACAAAAAGCCCACTCCAG-3' & AFP, forward & 147 & NM_001134 \\
OMC318 & 5'-GGTGCATACAGGAAGGGATG-3' & AFP, reverse & 147 & \\
OMC321 & 5'-CGAATGCCAGAGAAGGTCAC-3' & RPL19, forward & 157 & BC095445 \\
OMC322 & 5'-CCATGAGAATCCGCTTGTTT-3' & RPL19, reverse & 157 & \\
OMC755 & 5'-AGAAGGTGTGGGCAGAAGAA-3' & CD44, forward & 116 & BC004372 \\
OMC756 & 5'-AAATGCACCATTTCCTGAGA-3' & CD44, reverse & & \\
\hline
\end{tabular}

AFP, $\alpha$-fetoprotein; RPL19, ribosomal protein L19; CD44, cluster of differentiation 44.

was used in place of FBS to establish defined xenobiotic-free conditions and was added at a final concentration of $10 \%$.

Reverse transcription-quantitative polymerase chain reaction $(R T-q P C R)$. Cells were cultured in DMEM or HSM in 6-well plates. On days 0, 4 and 7, RNA was isolated using Isogen (Nippon Gene Co., Ltd., Tokyo, Japan). Total RNA (5 $\mu \mathrm{g})$ was subjected to cDNA synthesis using a SuperScript III FirstStrand Synthesis system (Thermo Fisher Scientific, Inc.), according to the manufacturer's protocol.qPCR was performed using Fast SYBR Green Master Mix (Thermo Fisher Scientific, Inc.) with the MiniOpticon (Bio-Rad Laboratories, Inc., Hercules, CA, USA) for 40 cycles consisted of two steps of denaturation at $95^{\circ} \mathrm{C}$ for $5 \mathrm{sec}$ and annealing-extension at $60^{\circ} \mathrm{C}$ for $5 \mathrm{sec}$. Table I lists the primer sequences used. Ribosomal protein L19 (RPL19), a constitutively expressed housekeeping gene, was used as an internal control (18). RPL19 was used as an endogenous control to monitor the amount of mRNA because the gene is a constitutively expressed housekeeping gene (18). The expression levels of the genes were analyzed automatically by the Mini Opticon system (Bio-Rad Laboratories, Inc.) based on delta-delta cycle quantification $(\Delta \Delta \mathrm{Cq})$ method (19). Relative expression level of a gene was calculated as expression level of a gene divided by expression level of RPL19.

Immunostaining. HLF and PLC/PRF/5 cells were spread onto 8-well chamber slides (Matsunami Glass Ind., Ltd., Kishiwada, Japan). The cells were cultured in DMEM or HSM. Following 7 days of culture, the cells were fixed with 4\% paraformaldehyde (Sigma-Aldrich; Merck KGaA, Darmstadt, Germany) for $30 \mathrm{~min}$ at $4^{\circ} \mathrm{C}$. Endogenous peroxidase was inactivated by incubation with $0.1 \%$ hydrogen peroxide in $100 \%$ methanol for $30 \mathrm{~min}$ at $4^{\circ} \mathrm{C}$. Specimens were incubated with $2 \%$ fetal bovine serum in PBS (wash buffer) at $4^{\circ} \mathrm{C}$ for $30 \mathrm{~min}$. Specimens were incubated with mouse anti-human AFP (catalog no. M225) (Takara Bio, Inc., Otsu, Japan) or mouse anti-human CD44 (catalog no. 3570s; Cell Signaling Technology, Inc., Danvers, MA, USA) antibody diluted 1:1,000 in wash buffer overnight at $4^{\circ} \mathrm{C}$. Specimens were rinsed with PBS three times, and incubated with a 1:1,000 dilution of horseradish peroxidase-labeled anti-mouse antibody (catalog no. NA934-100UL; GE Healthcare Bio-Sciences, Pittsburg, PA, USA) at $4^{\circ} \mathrm{C}$ for $3 \mathrm{~h}$. Diaminobenzidine (Dako; Agilent Technologies, Inc., Santa Clara, CA, USA) was applied, and the nuclei were stained with hematoxylin (Muto Pure Chemicals Co., Ltd., Tokyo, Japan) for 15 sec. Specimens were observed and images were captured using an AX80 photomicroscope (Olympus Corporation, Tokyo, Japan).

Statistical analysis. A one-way analysis of variance was applied for statistical analysis using JMP software (version 10.0.2; SAS Institute, Cary, NC, Inc.), and Tukey's test was used as a post hoc test. $\mathrm{P}<0.05$ was considered to indicate a statistically significant difference.

\section{Results}

To determine the effects of HSM on cell proliferation, the number of HLF (Fig. 1A) and PLC/PRF/5 (Fig. 1B) viable cells were determined following culture in DMEM or HSM. Viable cell numbers were observed to increase towards day 7 of culture in DMEM, whereas a decrease in viable cell number was observed towards day 7 of culture in HSM. Viable cell numbers were significantly increased following culture in DMEM compared with culture in HSM. These results suggest that HMS significantly decreased cell proliferation.

The mRNA expression levels of AFP (Fig. 2A) and CD44 (Fig. 2B) were analyzed using RT-qPCR. The expression levels of AFP and CD44 were significantly increased on day 7 in HLF and PLC/PRF/5 cells ( $\mathrm{P}<0.05$; Fig. 2). These results suggest that HSM upregulated the expression of AFP and CD44.

The expression levels of AFP and CD44 proteins were analyzed by immunostaining. The expression of AFP was identified to increase in HLF cells between day 0 (Fig. 3A) and day 7 (Fig. 3B), whereas its expression in PLC/PRF/5 cells did not appear to alter during this same timeframe (Fig. 3C and D). The expression level of CD44 was also increased in HLF cells between day 0 (Fig. 3E) and day 7 (Fig. 3F), but was not apparently altered in PLC/PRF/5 cells (Fig. 3G and H).

\section{Discussion}

Stem cells of HCC cloned with CD34 express AFP and CD44 (20). AFP is expressed by HCC cells through dedifferentiation (21). These studies suggest that HCC cells positive for AFP and CD44 are markers of cancer stem cells of HCC. In the present study, the expression levels of AFP and CD44 were identified to increase following incubation in HSM. To the best of our knowledge, there are currently no reported 

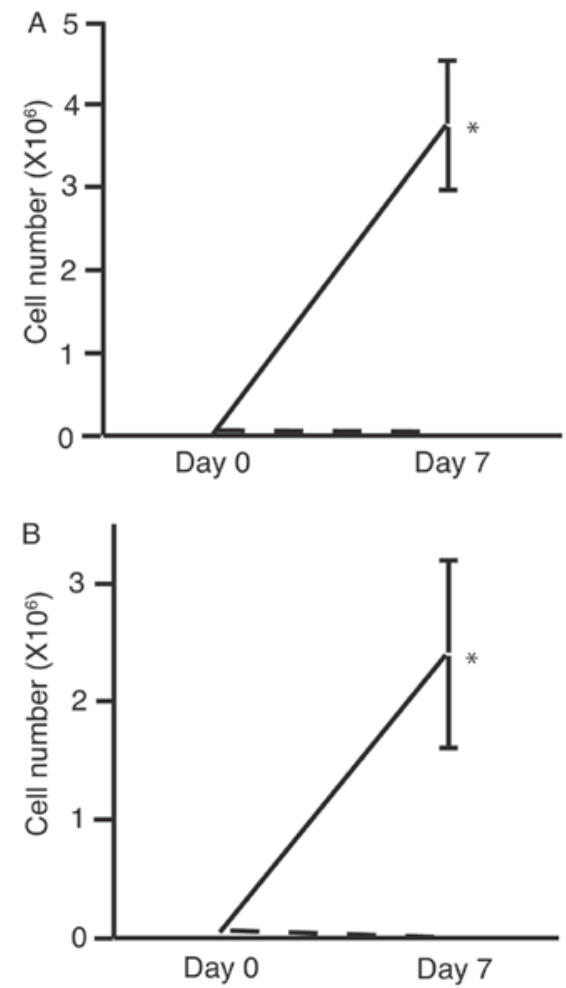

Figure 1. Viability of cells cultured in two separate media. (A) HLF and (B) PLC/PRF/5 cells were cultured in Dulbecco's modified Eagle's medium (unbroken line) or hepatocyte selection medium (broken line). Viability of cells was determined using a trypan blue assay at days 0 and 7 . ${ }^{*} \mathrm{P}<0.05$ vs. culture in HSM at day 7.
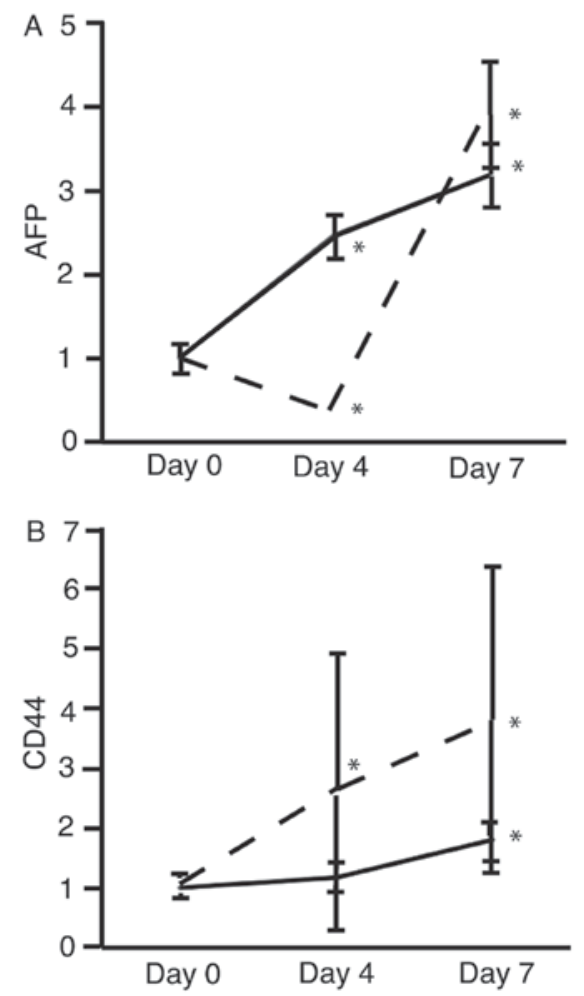

Figure 2. Expression levels of AFP and CD44. HLF (unbroken line) and PLC/PRF/5 (broken line) cells were cultured in hepatocyte selection medium. RNA was isolated and subjected to the reverse transcription-quantitative polymerase chain reaction to analyze the expression levels of (A) AFP and (B) CD44. ${ }^{*} \mathrm{P}<0.05$ vs. expression at day 0 . AFP, $\alpha$-fetoprotein; CD44, cluster of differentiation 44 .

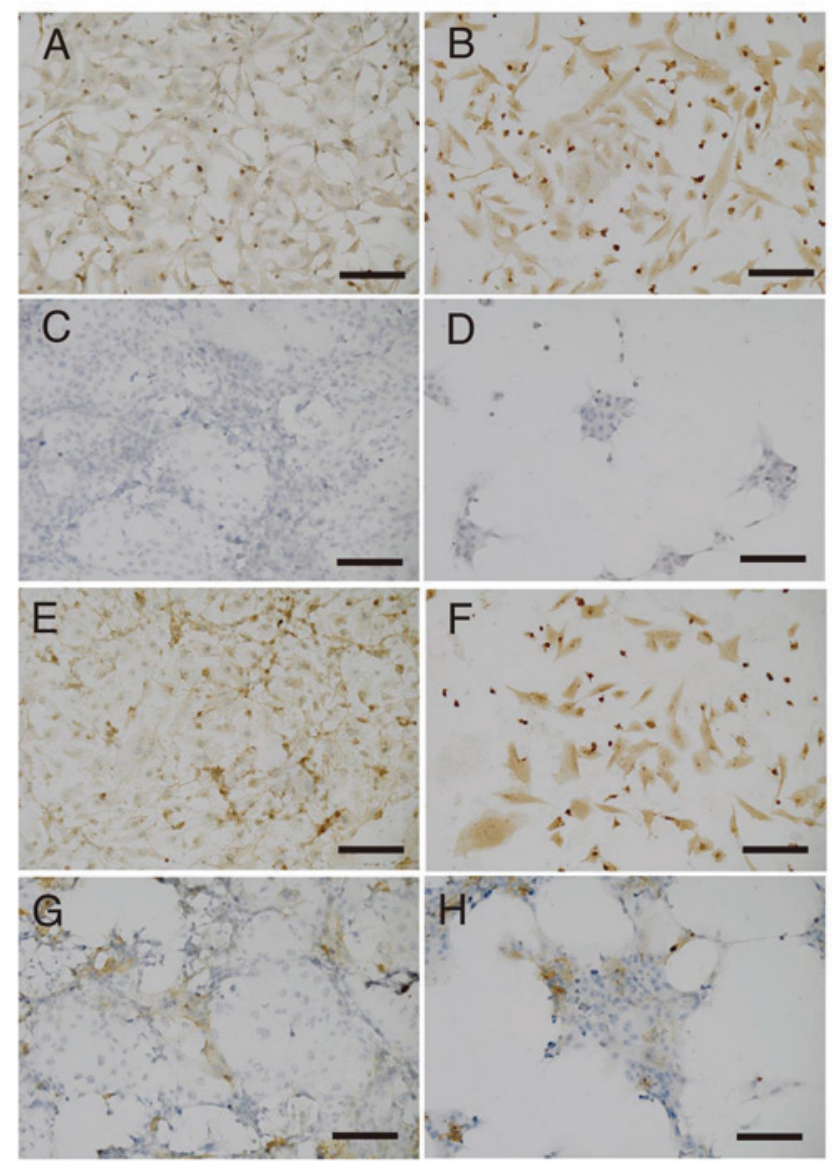

Figure 3. Immunostaining. HLF cells cultured in (A) DMEM or (B) HSM, or PLC/PRF/5 cells cultured in (C) DMEM or (D) HSM were subjected to immunostaining with an anti-AFP antibody. HLF cells cultured in (E) DMEM or (F) HSM, or PLC/PRF/5 cells cultured in (G) DMEM or (H) HSM were subjected to immunostaining with an anti-CD44 antibody. Scale bar, $100 \mu \mathrm{m}$. DMEM, Dulbecco's modified Eagle's medium; HSM, hepatocyte selection medium; AFP, $\alpha$-fetoprotein; CD44, cluster of differentiation 44.

studies on the culture of HCC cells in media lacking glucose. The biological significance of the results of the present study is unclear; however, there are two possibilities. One is that the transcriptional activity of the AFP and CD44 genes was activated. The other is that cancer stem cells were purified with HSM. To determine which hypothesis may be true, the promoter activities of AFP and CD44 should be analyzed. HCC cells positive for AFP exhibit the characteristics of cancer stem cells and are associated with poor prognosis $(22,23)$. The results of these previous studies and those of the present study suggest that HCC cells enriched in HSM have stem cell-like characteristics. Suppressing the expression of CD44 may be useful as a treatment for cells cultured in HSM (24).

The cancer microenvironment is typically lacking in glucose because the supply of glucose to the tumor is not sufficient $(25,26)$. In the present study, HCC cells were cultured in HSM, a medium deficient in glucose, to mimic the microenvironment of cancer. HSM was identified to be potentially useful to create an experimental cancer microenvironment model, such as fibrous tissues surrounding HCC (2). The results of the present study suggest that cancer stem cells from HCC were 
enriched in HSM, which is a potentially useful medium to enrich cancer stem cells from HCC.

A limitation of the present study was that the resultant cells cultured in HSM may not definitively be classified as stem cells. It is possible that these cells only have partial cancer stem cell characteristics.

In future studies, cells obtained from culture in HSM require propagation and investigation for cancer stem cell characteristics including analysis of cell-surface markers and spheroid formation assay $(27,28)$.

The results of the present study identified that HSM decreased the number of HCC cells, but the expression levels of AFP and CD44 were increased in the cells obtained from culture in HSM. Therefore, HSM is potentially useful for the enrichment of stem cells of HCC.

\section{Acknowledgements}

The present study was supported by a Grant-in-Aid for Scientific Research (C) from the Japan Society for the Promotion of Science (grant no. 15K09032).

\section{References}

1. Cameron AM: Screening for viral hepatitis and hepatocellular cancer. Surg Clin North Am 95: 1013-1021, 2015.

2. Tomizawa M, Kondo F and Kondo Y: Growth patterns and interstitial invasion of small hepatocellular carcinoma. Pathol Int 45: 352-358, 1995.

3. Leffert HL and Paul D: Studies on primary cultures of differentiated fetal liver cells. J Cell Biol 52: 559-568, 1972.

4. Ohira RH, Dipple KM, Zhang YH and McCabe ER: Human and murine glycerol kinase: Influence of exon 18 alternative splicing on function. Biochem Biophys Res Commun 331: 239-246, 2005.

5. Ai Y, Jenkins NA, Copeland NG, Gilbert DH, Bergsma DJ and Stambolian D: Mouse galactokinase: Isolation, characterization, and location on chromosome 11. Genome Res 5: 53-59, 1995.

6. Wheatley DN, Scott L, Lamb J and Smith S: Single amino acid (arginine) restriction: Growth and death of cultured HeLa and human diploid fibroblasts. Cell Physiol Biochem 10: 37-55, 2000.

7. Tomizawa M, Toyama Y, Ito C, Toshimori K, Iwase K, Takiguchi M, Saisho H and Yokosuka O: Hepatoblast-like cells enriched from mouse embryonic stem cells in medium without glucose, pyruvate, arginine, and tyrosine. Cell Tissue Res 333. 17-27, 2008.

8. Tomizawa M, Shinozaki F, Sugiyama T, Yamamoto S, Sueishi M and Yoshida T: Survival of primary human hepatocytes and death of induced pluripotent stem cells in media lacking glucose and arginine. PLoS One 8: e71897, 2013.

9. Ghosn MG, Tuchin VV and Larin KV: Depth-resolved monitoring of glucose diffusion in tissues by using optical coherence tomography. Opt Lett 31: 2314-2316, 2006.

10. Tomizawa M, Garfield S, Factor V and Xanthopoulos KG: Hepatocytes deficient in CCAAT/enhancer binding protein alpha (C/EBP alpha) exhibit both hepatocyte and biliary epithelial cell character. Biochem Biophys Res Commun 249: 1-5, 1998.

11. Tomizawa M, Wang YQ, Ebara M, Saisho H, Watanabe K, Nakagawara A and Tagawa M: Decreased expression of the CCAAT/enhancer binding protein alpha gene involved in hepatocyte proliferation in human hepatocellular carcinomas. Int $\mathbf{J}$ Mol Med 9: 597-600, 2002.
12. Guzman G, Alagiozian-Angelova V, Layden-Almer JE, Layden TJ, Testa G, Benedetti E, Kajdacsy-Balla A and Cotler SJ: p53, Ki-67, and serum alpha feto-protein as predictors of hepatocellular carcinoma recurrence in liver transplant patients. Mod Pathol 18: 1498-1503, 2005.

13. Orian-Rousseau V and Sleeman J: CD44 is a multidomain signaling platform that integrates extracellular matrix cues with growth factor and cytokine signals. Adv Cancer Res 123: 231-254, 2014.

14. Hiraga T, Ito $\mathrm{S}$ and Nakamura H: Cancer stem-like cell marker CD44 promotes bone metastases by enhancing tumorigenicity, cell motility and hyaluronan production. Cancer Res 73: 4112-4122, 2013

15. Kim R, Kim SB, Cho EH, Park SH, Park SB, Hong SK and Chae G: CD44 expression in patients with combined hepatocellular cholangiocarcinoma. Ann Surg Treat Res 89: 9-16, 2015.

16. Friemel J, Rechsteiner M, Frick L, Böhm F, Struckmann K, Egger M, Moch H, Heikenwalder M and Weber A: Intratumor heterogeneity in hepatocellular carcinoma. Clin Cancer Res 21: 1951-1961, 2015.

17. Nakamura T, Teramoto $\mathrm{H}$, Tomita $\mathrm{Y}$ and Ichihara A: L-proline is an essential amino acid for hepatocyte growth in culture. Biochem Biophys Res Commun 122: 884-891, 1984.

18. Davies B and Fried M: The L19 ribosomal protein gene (RPL19): Gene organization, chromosomal mapping, and novel promoter region. Genomics 25: 372-380, 1995.

19. Tam S, Clavijo A, Engelhard EK and Thurmond MC: Fluorescence-based multiplex real-time RT-PCR arrays for the detection and serotype determination of foot-and-mouth disease virus. J Virol Methods 161: 183-191, 2009.

20. Park SC, Zeng C, Tschudy-Seney B, Nguyen NT, Eun JR, Zhang Y, Ramsamooj R, Zhang Y, Zhao M, Theise ND, et al: Clonogenically culturing and expanding CD34+ liver cancer stem cells in vitro. Stem Cells Dev 24: 1506-1514, 2015.

21. Mu X, Español-Suñer R, Mederacke I, Affò S, Manco R, Sempoux C, Lemaigre FP, Adili A, Yuan D, Weber A, et al: Hepatocellular carcinoma originates from hepatocytes and not from the progenitor/biliary compartment. J Clin Invest 125: 3891-3903, 2015

22. Zhao X, Parpart S, Takai A, Roessler S, Budhu A, Yu Z, Blank M, Zhang YE, Jia HL, Ye QH, et al: Integrative genomics identifies YY1AP1 as an oncogenic driver in $\operatorname{EpCAM}(+) \operatorname{AFP}(+)$ hepatocellular carcinoma. Oncogene 34: 5095-5104, 2015.

23. Benzoubir N, Mussini C, Lejamtel C, Dos Santos A, Guillaume C, Desterke C, Samuel D, Bréchot C, Bourgeade MF and Guettier C: Gamma-smooth muscle actin expression is associated with epithelial-mesenchymal transition and stem-like properties in hepatocellular carcinoma. PLoS One 10: e0130559, 2015.

24. Gao Y, Ruan B, Liu W, Wang J, Yang X, Zhang Z, Li X, Duan J, Zhang F, Ding R, et al: Knockdown of CD44 inhibits the invasion and metastasis of hepatocellular carcinoma both in vitro and in vivo by reversing epithelial-mesenchymal transition. Oncotarget 6: 7828-7837, 2015.

25. Cheng GM and To KK: Adverse cell culture conditions mimicking the tumor microenvironment upregulate ABCG2 to mediate multidrug resistance and a more malignant phenotype. ISRN Oncol 2012: 746025, 2012.

26. Menendez JA, Oliveras-Ferraros C, Cufi S, Corominas-Faja B, Joven J, Martin-Castillo B and Vazquez-Martin A: Metformin is synthetically lethal with glucose withdrawal in cancer cells. Cell Cycle 11: 2782-2792, 2012.

27. Liao J, Qian F, Tchabo N, Mhawech-Fauceglia P, Beck A, Qian Z, Wang X, Huss WJ, Lele SB, Morrison CD and Odunsi K: Ovarian cancer spheroid cells with stem cell-like properties contribute to tumor generation, metastasis and chemotherapy resistance through hypoxia-resistant metabolism. PLoS One 9: e84941, 2014.

28. Zhu CP, Wang AQ, Zhang HH, Wan XS, Yang XB, Chen SG and Zhao HT: Research progress and prospects of markers for liver cancer stem cells. World J Gastroenterol 21: 12190-12196, 2015. 